\title{
Research Article The Classes of Mutual Compactificability
}

\author{
Martin Maria Kovár \\ Received 17 June 2004; Revised 28 November 2005; Accepted 13 February 2006 \\ Recommended by Lokenath Debnath
}

Two disjoint topological spaces $X, Y$ are mutually compactificable if there exists a compact topology on $K=X \cup Y$ which coincides on $X, Y$ with their original topologies such that the points $x \in X, y \in Y$ have disjoint neighborhoods in $K$. The main problem under consideration is the following: which spaces $X, Y$ are so compatible such that they together can form the compact space $K$ ? In this paper we define and study the classes of spaces with the similar behavior with respect to the mutual compactificability. Two spaces $X_{1}, X_{2}$ belong to the same class if they can substitute each other in the above construction with any space $Y$. In this way we transform the main problem to the study of relations between the compactificability classes. Some conspicuous classes of topological spaces are discovered as the classes of mutual compactificability. The studied classes form a certain "scale of noncompactness" for topological spaces. Every class of mutual compactificability contains a $T_{1}$ representative, but there are classes with no Hausdorff representatives.

Copyright (c) 2007 Martin Maria Kovár. This is an open access article distributed under the Creative Commons Attribution License, which permits unrestricted use, distribution, and reproduction in any medium, provided the original work is properly cited.

\section{The notation and terminology}

By a space we always mean a topological space. Throughout the paper we mostly use the standard topological notions as in [1] with the exception that all spaces are assumed without any separation axioms in general. Especially compactness and its modifications are understood without Hausdorff. Regarding the notions related to the Wallman compactification, our main source was [2]. Finally, we take from [3, 4] the terminology related to $\theta$-regularity. Let $S$ be a set. We denote by $|S|$ the cardinality of $S$. We will usually denote by $\tau$ or $\tau_{X}$ a topology of a space $X$ (in case that we will work simultaneously with more topological spaces). In a space $X$ a point $x \in X$ is in the $\theta$-closure of a set $A \subseteq X$ 
$\left(x \in \operatorname{cl}_{\theta} A\right)$ if every closed neighborhood of $x$ intersects $A$. The point $x$ belongs to the $\theta$-interior of $A\left(x \in \operatorname{int}_{\theta} A\right)$ if $x$ has a closed neighborhood $G \subseteq A$. The filter base $\Phi \theta$ converges to its $\theta$-limit $x$ if for every closed neighborhood $H$ of $x$ there is $F \in \Phi$ such that $F \subseteq H$. A net $\varphi(B, \geqslant$ ) has a $\theta$-cluster point (a $\theta$-limit) $x \in X$ if $x$ is a $\theta$-cluster point (a $\theta$-limit) of the derived filter base $\{\{\varphi(\alpha) \mid \alpha \geqslant \beta\} \mid \beta \in B\}$. We say that a space $X$ is (countably) $\theta$-regular if every (countable) filter base in $X$ with a $\theta$-cluster point has a cluster point. For more detailed characterization of $\theta$-regularity, the reader is referred to $[3,4]$. Recall that the preorder of specialization is a reflexive and transitive binary relation on $X$ defined by $x \leqslant y$ if and only if $x \in \operatorname{cl}\{y\}$. This relation is antisymmetric and hence is a partial ordering if and only if $X$ is a $T_{0}$ space. The points $x, y$ in a space $X$ are $T_{0}$-separable if there is an open set containing only one of the points $x, y$. The points $x, y$ are $T_{2}$-separable if they have open disjoint neighborhoods. In this notation, the space $X$ is said to be $S_{2}$ [2] if every two $T_{0}$-separable points of $X$ are $T_{2}$-separable. We say that the space $X$ is $R_{1}$ [3] if for every $x, y \in X$ satisfying $\operatorname{cl}\{x\} \neq \operatorname{cl}\{y\}$ the sets $\operatorname{cl}\{x\}, \operatorname{cl}\{y\}$ have disjoint neighborhoods. Obviously, the separation axioms $R_{1}$ and $S_{2}$ are equivalent and slightly weaker than Hausdorff. Let $X$ be a space. Two disjoint sets $A, B \subseteq X$ are said to be pointwise separated in $X$ if every $x \in A, y \in B$ are $T_{2}$-separable in $X$. In this paper we say that a space is (strongly) locally compact if its every point has a compact (closed) neighborhood. We denote by Slc the class of strongly locally compact spaces. A filter in a space $X$ is said to be ultraclosed (ultraopen, resp.) if it is maximal among all filters in $X$ having a base consisting of closed (open, resp.) sets [2]. By the Wallman compactification of $X$ we mean that the set $\omega X=X \cup\{y \mid y$ is a nonconvergent ultraclosed filter in $X\}$. The sets $\mathscr{S}(U)=U \cup\{y \mid y \in \omega X \backslash X, U \in y\}$, where $U$ is open in $X$, constitute an open base of $\omega X$ (see [2]).

\section{Preliminaries and introduction}

The class of compact Hausdorff spaces is widely accepted as one of the most important and best investigated classes of topological spaces. However, the situation completely changes if the Hausdorff separation axiom is omitted. Some topologists hold the view that non-Hausdorff spaces are less interesting-perhaps because some traditional mathematical disciplines, such as geometry or mathematical and functional analyses, very rarely offer important examples of non-Hausdorff topologies. But there is also another reason, sometimes acknowledged with some hesitation and reluctance. It is extremely difficult to handle a topological space, even with a compact one, and derive some deep results about it, if no separation is available. However, the latest developments in computer science motivated topology, domain theory, and also in modern physics lying beyond the standard model witness the importance of this way, apart from how difficult and troublesome it can sometimes be.

The interesting paper of Herrlich [5] is one of the opening papers which systematically studies the notion of non-Hausdorff compactness and contains really nontrivial results. In this paper, Herrlich introduces a useful gradation of compactness. A space $X$ is said to be $k$-complete provided that any filter on $X$, which contains an element of each cover of $X$ by less than $k+1$ open sets, converges in $X$, where $k=1,2, \ldots, \omega$. It is not difficult to show that every compact Hausdorff space is 2-complete and the compactness itself is 
equivalent to $\omega$-completeness. In this way the compact spaces may be classified by their "degree of compactness," represented by $k$-completeness. The study of the properties of compactifications (and, of course, of $k$-completions of [5]) touches the problem from another side. For instance, in [6], Hušek proved that the well-known reflection properties of the Čech-Stone compactification have no analogue in the realm of non-Hausdorff spaces-there exist spaces having no (weak) reflection in compact spaces (for the precise definition, see [6] or [7]). This result was improved and generalized in [7]: a space has a weak reflection in compact spaces if and only if its growth in the Wallman compactification is finite. The properties of the growth of a noncompact space in some compactification may carry important information about the space and "how far" it is from compactness. Besides this, there exist scientific papers which study a rather fascinating question: when is it possible to replace a growth of a space in some compactification by another space? Some preliminary results are well known and reader can find a brief introduction to this topic, at least for locally compact spaces, for instance, in [1]. Unfortunately, the role of a space and its growth is strongly nonsymmetric, which sometimes may be viewed as a disadvantage. Therefore, it is very natural to study also the symmetrical case.

In this paper we will continue evolving the idea of mutual compactificability, introduced in the previous paper [8]. The following simple fact, proved already in [9], was the main source of inspiration for writing the paper [8]: if $X$ is a $\theta$-regular space, then $X$ and $\omega X \backslash X$ are pointwise separated in $\omega X$. Then $X$ may be considered as a fragment of some compact space. But the things also work in the reverse way: let $K=X \cup Y$ be a compact space, which is a disjoint union of subspaces $X, Y$, such that every two points $x \in X, y \in Y$ have open disjoint neighborhoods in $K$. Then both $X$ and $Y$ are $\theta$-regular. An alternative approach to $\theta$-regularity is to consider it as a special kind of a separation axiom - the points can be separated from the closed filter bases having no cluster point by open disjoint neighborhoods [4]. Thus taking $\theta$-regularity as a tool for investigating the non-Hausdorff compactness is a certain compromise between the requirement of a sufficient level of generality and the convenience of leaving some kind of separation, latently contained in every compact space, still as a part of the game.

Now, let us recall the precise definition of mutual compactificability.

Definition 2.1. Let $X, Y$ be spaces with $X \cap Y=\varnothing$. The space $X$ is said to be compactificable by the space $Y$ or, in other words, $X, Y$ are called mutually compactificable if there exists a compact topology on $K=X \cup Y$ extending the topologies of $X$ and $Y$ such that the sets $X, Y$ are pointwise separated in $K$. Let $X$ and $Y$ be disjoint and mutually compactificable. Then any compact topology on $K=X \cup Y$ which induces the original topologies on $X, Y$ such that $X, Y$ are in $K$ pointwise separated is called $\mathscr{C}$-acceptable for the spaces $X, Y$.

Note that taking some additional conditions for the topology of the space $K$ in Definition 2.1 we can modify the notion of mutual compactificability in a natural and sensible way. For instance, we may request $K$ to be Hausdorff, connected, manifold, and so forth. In this way we can obtain the notions of $T_{2}$-mutually compactificable spaces, connectedly mutually compactificable spaces, manifold mutually compactificable spaces, and so forth. The reader can find in a few theorems and examples of [8] some very initial 
comparison of the properties of the mutual compactificability and its $T_{2}$ modification. However, a more detailed and complex study of these modifications will be presented in some future paper(s). Now, let us summarize some of the most important results of [8] and take them as our starting point. For the proofs, the reader is referred to [8].

Theorem 2.2 (see [8]). The following statements hold.

(i) For a space $X$ there exists a space $Y$ such that $X, Y$ are mutually compactificable if and only if $X$ is $\theta$-regular.

(ii) If $X$ is a regular space on which every continuous real-valued function is constant, then $X$ is compactificable by no $S_{2}$ space.

(iii) Any two disjoint strongly locally compact spaces are mutually compactificable.

(iv) If $X$ is compactificable by some compact space $Y$, then $X$ is strongly locally compact.

Note that the class of spaces considered in (ii) yields another argument for studying $\theta$ regular spaces and the mutual compactificability. Regular spaces, as well as the properties of various compactifications and their growths, are widely accepted as legitimate objects of study. Thus consider $Y=\omega X \backslash X$, where $X$ belongs to class mentioned above. It is a $\theta$-regular space which is not regular, and, moreover, it can be replaced by no Hausdorff or regular space. This fact is completed by two other observations, recapitulated in the following example.

Example 2.3 (see [8]). The following statements hold.

(i) There exists a regular non- $T_{3.5}$ space which is compactificable by the infinite countable discrete space.

(ii) There exists a nonlocally compact space $T_{2}$-compactificable by a strongly locally compact space.

\section{The classes of mutual compactificability}

Having two general topological spaces, it might be a difficult problem to determine whether their disjoint union admits any $\mathscr{C}$-acceptable topology (we already know that in such a case, both of the spaces must be at least $\theta$-regular). However, the situation could be easier if we know that the behavior of these spaces is, in some way, similar to that of the spaces which are more familiarly known, which can be simply constructed, and which satisfy, if possible, some stronger separation axioms. This considerations naturally lead to the following definition.

Definition 3.1. Let Top be the class of all topological spaces. For any $X, Z \in$ Top, define $X \sim Z$ if for every nonempty space $Y \in$ Top disjoint from the spaces $X, Z$ the space $X$ is compactificable by $Y$ if and only if $Z$ is compactificable by $Y$. It can be easily seen that is reflexive, symmetric, transitive, and hence it is an equivalence relation. Denote by $\mathscr{C}(X)$ the equivalence subclass of Top with respect to $\sim$ containing $X$ and call it the compactificability class of $X$. Now, for any $X, Z \in$ Top, put $\mathscr{C}(X) \succcurlyeq \mathscr{C}(Z)$ if for every nonempty space $Y \in$ Top, the following holds: if the space $X$ is compactificable by $Y$ disjoint from the spaces $X, Z$, then $Z$ is compactificable by $Y$. Obviously, the relation $\succcurlyeq$ is reflexive, antisymmetric, transitive, and hence it is an order relation between the compactificability 
classes. If for some $X, Z \in$ Top it holds $\mathscr{C}(X) \succcurlyeq \mathscr{C}(Z)$ but $\mathscr{C}(X) \neq \mathscr{C}(Z)$, write $\mathscr{C}(X) \succ$ $\mathscr{C}(Z)$.

One of the aims of the presented theory is to give a description of the compactificability classes of spaces which are relatively familiar-for example, the spaces constructed from the real line, the Cantor and Tykhonov cubes, and so forth. But to be able to do it, we need some more general results, which we will present in the current paper. The author will present the results which are more specific and concern the spaces mentioned above, in the next two forthcoming papers.

The following theorem is quite clear and so we can omit its proof.

Theorem 3.2. The spaces $X, Z$ are mutually compactificable if and only if any two representatives $P \in \mathscr{C}(X), Q \in \mathscr{C}(Z)$ are mutually compactificable. In particular, the space $X$ is compactificable by a copy of itself if and only if the class $\mathscr{C}(X)$ contains two mutually compactificable representatives.

In this connection we can speak about mutually compactificable classes. The fact mentioned in the following example is an immediate consequence of (iii) of Theorem 2.2.

Example 3.3. For any two strongly locally compact spaces $X, Z$, the classes $\mathscr{C}(X), \mathscr{C}(Z)$ are mutually compactificable.

Remark that the analogous notions of $T_{2}$-compactificability classes, connected compactificability classes, or manifold compactificability classes may be also very naturally defined and studied. It is an important question whether there exists more than one different classes and whether at least one of the classes contains more than one homeomorphism subclasses. To prove it, the following lemma will be useful.

Lemma 3.4. Let $X$ be a $\theta$-regular space. Suppose that there exists a space $Z$ with $\mathscr{C}(X) \succcurlyeq$ $\mathscr{C}(Z)$. Then for every $z \in Z$, there is a compact set $K_{z} \subseteq X$ such that $X=\bigcup_{z \in Z} K_{z}$.

Proof. Let $C$ be any compactification of $X$ such that $X$ and $F=C \backslash X$ are pointwise separated in $C$. Since $X$ is $\theta$-regular, such compactification always exists; one can take $C=\omega X$ for example. Denote $J=[0,1]$ and $J_{0}=[0,1)$ and consider these real intervals with the Euclidean topology. We put $K=J \times C, Y=K \backslash(\{1\} \times X)$. The spaces $Y$ and $\{1\} \times X$ are pointwise separated and $\{1\} \times X$ is homeomorphic to $X$. Hence, the product topology $\tau_{K}$ on $K$ is $\mathscr{b}$-acceptable for the spaces $\{1\} \times X$ and $Y$. For every $x \in X, t \in J_{0}$, we define $\zeta_{x}(t)=(t, x)$. Consider the net $\zeta_{x}\left(J_{0}, \geqslant\right)$. It converges to $(1, x)$ for every $x \in X$, but it has no cluster point in $Y$ since the spaces $\{1\} \times X$ and $Y$ are pointwise separated.

Without loss of generality, we may assume that $Y \cap Z=\varnothing$ (otherwise, one can take another homeomorphic copy of $Z$ disjoint from $Y)$. Since $\mathscr{C}(X) \succcurlyeq \mathscr{C}(Z)$ by the assumption, there exists some $\mathscr{C}$-acceptable topology $\tau_{L}$ on $L=Y \cup Z$. For every $z \in Z$, we put $M_{z}=\left\{x \mid x \in X, \zeta_{x}\right.$ has a cluster point $z$ in $\left.L\right\}$. Notice that

$$
X=\bigcup_{z \in Z} M_{z}
$$

since for every $x \in X$ the net $\zeta_{x}$ has a cluster point in the compact space $L$. We will show that every $M_{z}$ is has a compact closure in $X$. 
Suppose that there exists a net $\varphi(A, \geqslant)$ with values in $M_{z}$ and a cluster point $l \in F$. Let $W_{L} \in \tau_{L}$ be any open neighborhood of $(1, l) \in Y$. The set $W_{L} \cap Y$ is open in $Y$ and so there exists $W_{K} \in \tau_{K}$ such that $W_{L} \cap Y=W_{K} \cap Y$. Since $K=J \times C$ there are $(a, 1] \in \tau_{J}$, $U \in \tau_{C}$ such that $(1, l) \in(a, 1] \times U \subseteq W_{K}$. It follows that there exists some $\beta \in A$ such that $\varphi(\beta) \in U$. Now, let $V \in \tau_{L}$ be such that $z \in V$. We put $x=\varphi(\beta)$. Then $x \in M_{z}$ and so $\zeta_{x}\left(J_{0}, \geqslant\right)$ has a cluster point $z$. Obviously, there exists $t \in J_{0}$ such that $t>a$ and $\zeta_{x}(t) \in$ $V$. But $\zeta_{x}(t)=(t, \varphi(\beta)) \in(a, 1] \times U \subseteq W_{K}$. On the other hand, $\zeta_{x}(t) \in Y$ and so $\zeta_{x}(t) \in$ $W_{K} \cap Y=W_{L} \cap Y \subseteq W_{L}$. It follows that $W_{L} \cap V \neq \varnothing$. Hence, the points $(1, l) \in Y$ and $z \in Z$ have no disjoint neighborhoods in $L$, which contradicts to the assumption that the topology $\tau_{L}$ is $\mathscr{C}$-acceptable. Hence, no net with values in $M_{z}$ has a cluster point in $F$, which implies that $\mathrm{cl}_{\mathrm{C}} M_{z} \subseteq X$ and obviously, $K_{z}=\mathrm{cl}_{\mathrm{X}} M_{z}=\mathrm{cl}_{\mathrm{C}} M_{z}$ is compact.

Theorem 3.5. Let $X, Z$ be infinite discrete spaces with the cardinalities $|Z|<|X|$. Then $\mathscr{b}(Z) \succ \mathscr{b}(X)$.

Proof. Without loss of generality, we may assume that $Z \subsetneq X$. Firstly, suppose that there is some space $P \neq \varnothing, P \cap X=\varnothing$ which is compactificable by $Z$. Then there exists a $\mathscr{C}$ acceptable topology on $M=P \cup Z$, say $\tau_{M}$. We denote $S=X \backslash Z$. Let $p \in P$ be a point and let $\sigma$ be a family consisting exactly of the following sets:

(1) $U \in \tau_{M}$ for $p \notin U$,

(2) $U \cup(S \backslash F)$ for $p \in U \in \tau_{M}$ and $F \subseteq S$ finite,

(3) $\{x\}$ for $x \in S$.

We will show that $\sigma$ is a base of some $\mathscr{C}$-acceptable topology on $N=P \cup X$. One can easily check that $\sigma$ is closed under finite intersections and covers $N$, so it is a base of some compact topology $\tau_{N}$ on $N$. Obviously, the sets $P$ and $X$ are pointwise separated, the topology on $M$ induced from $N$ coincides with $\tau_{M}$ and the singletons of $S$ are open in $\tau_{N}$. Let $x \in Z$. Since $P$ and $Z$ are pointwise separated, there is some $U \in \tau_{M}$ such that $x \in U$ but $p \notin U$. Then $U \in \tau_{N}$ and hence, $\{x\}=U \cap X$ is open in the topology of $X$ induced from $N$. Hence, the induced topology of $X$ is discrete, which means that $\tau_{N}$ is $\mathscr{C}$ acceptable for the spaces $P, X$. Then $\mathscr{C}(Z) \succcurlyeq \mathscr{C}(X)$. Now, suppose that also $\mathscr{C}(X) \succcurlyeq \mathscr{C}(Z)$. In the notation of Lemma 3.4, the sets $K_{z}$ are finite since $\tau_{X}$ is discrete. Then $X$ is covered by $|Z|$ many finite sets, which is impossible. Hence, it follows $\mathscr{C}(Z) \succ \mathscr{C}(X)$.

Corollary 3.6. The compactificability classes do not form a set.

The next two results are immediate or easy consequences of Theorem 2.2. However, we should state them because of completeness. At least, they can point out that there exist compactificability classes containing more than one homeomorphism subclasses.

THeorem 3.7. Non- $\theta$-regular spaces form a compactificability class.

We call the class of non- $\theta$-regular spaces improper because no element of that class is compactificable by any space, wherefore this theory can say nothing more regarding such spaces. We will call other classes proper. Another important compactificability class is the class Comp of all compact spaces.

Theorem 3.8. The class Comp forms a class of compactificability. 
Proof. Indeed, let $X$ be compact and suppose that $X$ is compactificable by some space $Z$. Then $Z$ is strongly locally compact by Theorem 2.2(iv). If $Y$ is a strongly locally compact space, then by Theorem 2.2(iii), $Y$ is compactificable by $Z$. Hence, $\mathscr{C}(X) \succcurlyeq \mathscr{C}(Y)$. In particular, for a compact space $Y$, it follows $\mathscr{C}(X)=\mathscr{C}(Y)$.

Theorem 3.9. Let $Z$ be a noncompact $\theta$-regular space. Then $\mathscr{C}(Z) \neq$ Comp. If $Z$ is in addition locally compact, then Comp $\succ \mathscr{C}(Z)$.

Proof. Since $Z$ is $\theta$-regular, $Z$ and $\omega Z \backslash Z$ are pointwise separated (see [9] or [8]). Let $I=[0,1], J=(0,1]$. We put $L=I \times \omega Z, Y=L \backslash(\{0\} \times Z)$. Then $Y$ and $\{0\} \times Z$ are pointwise separated and $\mathscr{C}(Z)=\mathscr{C}(\{0\} \times Z)$. Suppose that $\mathscr{C}(Z) \succcurlyeq \mathscr{C}(X)$ for some compact $X$ disjoint from $Y$. Then there exists a $\mathscr{C}$-acceptable topology on $K=X \cup Y$, say $\tau_{K}$. Let $\varphi(A, \geqslant)$ be a net in $Z$ with a limit $z \in \omega Z \backslash Z$. For every $\alpha \in A, t \in J$, let $\psi_{\alpha}(t)=$ $(t, \varphi(\alpha))$. It is clear that $(0, \varphi(\alpha))$ is a limit of the net $\psi_{\alpha}(J, \leq)$. Since $Y$ and $\{0\} \times Z$ are pointwise separated, it follows that $\psi_{\alpha}(J, \leq)$ has no cluster point in $Y$. Since $K$ is compact, $\psi_{\alpha}(J, \leq)$ has a cluster point $\xi(\alpha) \in X$. But $X$ is compact and so the net $\xi(A, \geqslant)$ has a cluster point $x \in X$. Since $X$ and $Y$ are pointwise separated, there are $U, V \in \tau_{K}$ such that $x \in U$, $(0, z) \in V$, and $U \cap V=\varnothing$. The set $V \cap Y$ is open in $Y$ and so there exists $W \in \tau_{L}$ such that $V \cap Y=W \cap Y$. Then $(0, z) \in W$ and hence, there is some $\alpha \in A$ such that for every $\beta \geqslant \alpha$, we have $(0, \varphi(\beta)) \in W$. Then, there exists $t_{\beta} \in J$ such that for $0<t \leq t_{\beta}$, it follows $\psi_{\beta}(t) \in W \cap Y \subseteq V$. Choose $\beta \in A, \beta \geqslant \alpha$ such that $\xi(\beta) \in U$. There is some $t \in\left(0, t_{\beta}\right]$ with $\psi_{\beta}(t) \in U$. Then $\psi_{\beta}(t) \in U \cap V$, which is a contradiction. It follows $\mathscr{C}(Z) \neq \mathscr{C}(X)$.

Let $Z$ be locally compact. Then $Z$ is strongly locally compact since it is $\theta$-regular. Using the same consideration as in the proof of the previous theorem, we obtain $\mathscr{C}(X) \succcurlyeq \mathscr{C}(Z)$. Hence, $\mathscr{b}(X) \succ \mathscr{b}(Z)$.

Corollary 3.10. The class Comp is a maximal element in $\succcurlyeq$ among all proper classes of compactificability.

On proper classes, the relation $\succcurlyeq$ can be interpreted as a scale for various kinds of "noncompactness." If $\mathscr{C}(X) \succcurlyeq \mathscr{C}(Z)$ for $\theta$-regular spaces $X, Z$, then the space $X$ can be considered to be closer to be compact than the space $Z$. This works best for strongly locally compact spaces since among their classes the class Comp is the greatest element. The compactificability class of a nonstrongly locally compact space may be incomparable with Comp.

Lemma 3.11. Let $C$ be a compact $T_{2}$ space containing a dense nonopen subspace $X \subseteq C$. Then $\mathscr{C}(X)$ and $\mathscr{C}(Z)$ are comparable for no strongly locally compact $Z$.

Proof. We put $K=I \times C$, where $I=[0,1]$ has the Euclidean topology, $Y=K \backslash(\{0\} \times X)$. Suppose that $Z$ is a strongly locally compact space such that $Y \cap Z=\varnothing$ and $\mathscr{C}(X) \succcurlyeq$ $\mathscr{C}(Z)$. The space $\{0\} \times X$ is homeomorphic to $X$, so we also have $\mathscr{C}(\{0\} \times X) \succcurlyeq \mathscr{C}(Z)$. The product topology $\tau_{K}$ on $K$ is $\mathscr{C}$-acceptable for the spaces $\{0\} \times X, Y$ since $K$ is Hausdorff. Then there exists also some $\mathscr{C}$-acceptable topology on $L=Y \cup Z$, say $\tau_{L}$. We will show that $\{0\} \times(C \backslash X) \subseteq \mathrm{cl}_{\mathrm{L}} Z \backslash Z$.

Let $y \in C \backslash X$. Since $X$ is dense in $C$, there is a net $\varphi_{y}(A, \geqslant)$ with values in $X$ converging to $y$. Denote $J=(0,1]$. For every $\alpha \in A, t \in J$, let $\psi_{\alpha}(t)=\left(t, \varphi_{y}(\alpha)\right)$. The net $\psi_{\alpha}(J, \leq)$ 
has a limit $\left(0, \varphi_{y}(\alpha)\right) \in\{0\} \times X$ which is its unique cluster point in the Hausdorff space $K$. In particular, $\psi_{\alpha}(J, \leq)$ has no cluster point in $Y$. Since $L$ is compact, it follows that $\psi_{\alpha}(J, \leq)$ has a cluster point $\zeta(\alpha) \in Z$. Let $z \in Z$ be a cluster point of the net $\zeta(A$, $\geqslant)$. Suppose that $z \neq(0, y)$. Since $Y$ is $T_{2},(0, y) \in Y$, and $Y, Z$ are pointwise separated in $L$, there exist $U, V \in \tau_{L}$ such that $z \in U,(0, y) \in V$ and $U \cap V \cap Y=\varnothing$. The set $V \cap Y$ is open in $Y$, so there is $W \in \tau_{K}$ such that $V \cap Y=W \cap Y$. Then $(0, y) \in W$, which implies that there exists $\alpha \in A$ such that $\left(0, \varphi_{y}(\beta)\right) \in W$ for every $\beta \geqslant \alpha$. But then there exists $t_{\beta} \in J$ such that for every $0<t \leq t_{\beta}$, it follows $\psi_{\beta}(t) \in W \cap Y=V \cap Y$. Let us take $\beta \in A, \beta \geqslant \alpha$ such that $\zeta(\beta) \in U$. There exists $t \in\left(0, t_{\beta}\right]$ such that $\psi_{\beta}(t) \in U$, which contradicts $U \cap V \cap Y=\varnothing$. Hence, $z=(0, y)$ and so, $(0, y) \in \mathrm{cl}_{\mathrm{L}} Z \backslash Z$. It follows $\{0\} \times(C \backslash X) \subseteq \mathrm{cl}_{\mathrm{L}} Z \backslash Z$.

It follows that $Z$ and $\mathrm{cl}_{\mathrm{L}} Z \backslash Z$ are pointwise separated in the compact space $\mathrm{cl}_{\mathrm{L}} Z$. Since $Z$ is strongly locally compact, it follows that $Z$ is an open subspace of $\mathrm{cl}_{\mathrm{L}} Z, \mathrm{cl}_{\mathrm{L}} Z \backslash Z$ is closed in $\mathrm{cl}_{\mathrm{L}} Z$ and hence compact. Further, one can easily check that $\{0\} \times(C \backslash X)$ is a closed subspace of $Y$. Because $\{0\} \times(C \backslash X) \subseteq \operatorname{cl}_{\mathrm{L}} Z \backslash Z \subseteq Y$, it follows that $\{0\} \times(C \backslash$ $X)$ is a closed subspace of $\mathrm{cl}_{\mathrm{L}} Z \backslash Z$ and hence compact. Then $C \backslash X$ is compact as well and, consequently, closed in the Hausdorff space $C$. Then $X$ is an open subspace of $C$, which is a contradiction. Hence, $\mathscr{b}(X) \neq \mathscr{C}(Z)$. Suppose that $\mathscr{b}(Z) \succcurlyeq \mathscr{b}(X)$. Then $X$ is compactificable by a singleton, which is impossible since $X$ is not locally compact. It follows $\mathscr{C}(Z) \neq \mathscr{C}(X)$ as well.

As an immediate consequence of the previous lemma, we obtain the following theorem.

Theorem 3.12. Let $X$ be a $T_{3.5}$ space which is not locally compact and let $Z$ be a strongly locally compact (not necessarily $T_{2}$ ) space. Then $\mathscr{C}(X)$ and $\mathscr{C}(Z)$ are not comparable in the order $\succcurlyeq$.

The study of some compactificability class could be easier if the class contains a representative satisfying some separation axiom. Unfortunately, there exist compactificability classes with no Hausdorff representatives. As we already mentioned above, the next result follows directly from Theorem 2.2(ii).

Corollary 3.13. Let $X$ be a regular space on which every continuous real-valued function is constant. Then $\mathscr{C}(\omega X \backslash X)$ contains no Hausdorff representative.

On the other hand, it can be proved that every proper compactificability class contains a $T_{1}$ representative. Of course, the improper class contains even Hausdorff representatives - the $T_{2}$ spaces which are not regular. Let $X$ be a space. We say that $Y \subseteq X$ is a minimal subspace of $X$, if every element of $Y$ is minimal in the preorder of specialization of $X$ and every element of $X$ is comparable with some element of $Y$. More precisely, $Y \subseteq X$ is a minimal subspace of $X$ if and only if the following conditions are satisfied.

(1) If $y \in Y$ and $x \leqslant y$ for some $x \in X$, then $y \leqslant x$.

(2) If $x \in X$, then there is $y \in Y$ with $y \leqslant x$.

It can be easily seen that if $X$ is $T_{0}$ and $Y$ is a minimal subspace of $X$, then every singleton $\{y\}$, where $y \in Y$, is closed in $X$ and, consequently, $Y$ is $T_{1}$ in the induced topology. 
Lemma 3.14. Let $X$ be a $\theta$-regular space. Then $X$ contains a minimal subspace which is $T_{1}$ in the induced topology.

Proof. At first, suppose that $X$ is $T_{0}$. Then the preorder $\leqslant$ of specialization of $X$ is antisymmetric and hence is an order on $X$. Let $M \subseteq X$ be any linearly ordered chain with respect to $\leqslant$. Pick some fixed element $x \in M$ and take any closed neighborhood $G$ of $x$. For any $y \in M, y \leqslant x$, it follows $y \in \operatorname{cl}\{x\} \subseteq G$. Hence, the net $\operatorname{id}_{M}(M, \leqslant) \theta$-converges to $x$. Since $X$ is $\theta$-regular, $\operatorname{id}_{M}(M, \leqslant)$ has a cluster point, say $z \in X$. Let $U$ be an open neighborhood of $z$ and $t \in M$. There is some $s \in M$ such that $s \leqslant t$ and $s \in U$ since $z$ is a cluster point of $\operatorname{id}_{M}(M, \leqslant)$. Since $s \in \operatorname{cl}\{t\}$, we have $t \in U$, which means that $z \in \operatorname{cl}\{t\}$. Hence, $z \leqslant t$. Therefore, $z$ is a lower bound of $M$. By Zorn's lemma, every element of $X$ is comparable with some minimal element with respect to $\leqslant$. Let $Y$ be the set of all minimal elements of $X$. Then $Y$ is a minimal subspace of $X$, which is $T_{1}$ in the induced topology.

If $X$ is not $T_{0}$, we put $x \sim y$ if and only if $x \leqslant y$ and $y \leqslant x$ for every $x, y \in X$. The relation $\sim$ is an equivalence relation on $X$. Let $X_{0} \subseteq X$ be a subspace of $X$ such that the intersection of $X_{0}$ with every equivalence class is a singleton. The points that belong to the same equivalence class have the same open neighborhoods in $X$. Hence, $X_{0}$ is $\theta$-regular and is $T_{0}$, so by the previous paragraph, there is a minimal subspace $Y$ of $X_{0}$ which is $T_{1}$ in the induced topology. It is easy to check that $Y$ is also a minimal subspace of $X$.

Theorem 3.15. Every proper compactificability class contains a $\theta$-regular $T_{1}$ representative.

Proof. Let $X$ belong to some proper compactificability class. Then $X$ is $\theta$-regular and by Lemma 3.14, there exists a minimal $T_{1}$ subspace $X_{1} \subseteq X$. We will show that $\mathscr{C}(X)=$ $\mathscr{C}\left(X_{1}\right)$.

Suppose that there is some $Y$ with $X \cap Y=\varnothing$ which is compactificable by $X_{1}$. Let $\tau_{K_{1}}$ be any $\mathscr{C}$-acceptable topology on $K_{1}=X_{1} \cup Y$ with respect to $X_{1}, Y$. We put $K=X \cup Y$ and $\tau_{K}=\left\{W \mid W \subseteq K, W \cap K_{1} \in \tau_{K_{1}}, W \cap X \in \tau_{X}\right\}$. It is easy to check that $\tau_{K}$ is a topology on $K$. Let $\tau_{K / X}, \tau_{K / K_{1}}$, and $\tau_{K / Y}$ be the corresponding induced topologies on $X, K_{1}$, and $Y$. It is clear that $\tau_{K / X} \subseteq \tau_{X}$ and $\tau_{K / K_{1}} \subseteq \tau_{K_{1}}$. Conversely, let $U \in \tau_{K_{1}}$. Then $U \cap X_{1} \in \tau_{X_{1}}$ and so there exists $V \in \tau_{X}$ such that $U \cap X_{1}=V \cap X_{1}$. We put $W=(U \cap Y) \cup V$. Then $W \cap K_{1}=\left(U \cap Y \cap K_{1}\right) \cup\left(V \cap K_{1}\right)=(U \cap Y) \cup\left(V \cap X \cap K_{1}\right)=(U \cap Y) \cup\left(V \cap X_{1}\right)=$ $(U \cap Y) \cup\left(U \cap X_{1}\right)=U \cap\left(Y \cup X_{1}\right)=U \cap K_{1}=U \in \tau_{K_{1}}$. Further, $W \cap X=V \cap X=$ $V \in \tau_{X}$. Hence, $W \in \tau_{K}$ and then $U \in \tau_{K / K_{1}}$. It follows that $\tau_{K / K_{1}}=\tau_{K_{1}}$ and, consequently, $\tau_{K / Y}=\tau_{Y}$. Let $V \in \tau_{X}$. Then $V \cap X_{1} \in \tau_{X_{1}}$, so there exists $U \in \tau_{K_{1}}$ such that $V \cap X_{1}=$ $U \cap X_{1}$. Again, we put $W=(U \cap Y) \cup V$. Similarly as at the previous step, $W \in \tau_{K}$ and $V=W \cap X$, which gives $V \in \tau_{K / X}$. Then $\tau_{K / X}=\tau_{X}$. We will show that $K$ is compact. Let $\Omega$ be a $\tau_{K}$-open cover of $K$. Since $K_{1}$ is a compact subspace of $K$, there are $U_{1}, U_{2}, \ldots, U_{k} \in \Omega$ such that $K_{1} \subseteq \bigcup_{i=1}^{k} U_{i}$. Let $x \in K \backslash K_{1}=X \backslash X_{1}$. There exists $x_{1} \in X_{1}$ such that $x_{1} \in \operatorname{cl}_{X}\{x\}$. Also, there is some $i=1,2, \ldots, k$ such that $x_{1} \in U_{i}$. But then $x \in U_{i}$ which means that $K \subseteq \bigcup_{i=1}^{k} U_{i}$. Hence, $K$ is compact. Finally, we will show that $X, Y$ are pointwise separated in $K$. Let $x \in X, y \in Y$. Firstly, we will construct such open sets $U, V, P \in \tau_{K}$ such that

(1) $x \in V \subseteq U$,

(2) $y \in P$, 
(3) $\operatorname{cl}_{X}(V \cap X) \subseteq U$,

(4) $P \cap U \cap K_{1}=\varnothing$.

For every $t \in X_{1}$, there are $Q_{t}, S_{t} \in \tau_{K_{1}}$ such that $y \in Q_{t}, t \in S_{t}$, and $Q_{t} \cap S_{t}=\varnothing$ since $X_{1}$ and $Y$ are pointwise separated in $K_{1}$. Then, for every $t \in X_{1}$, there exist $P_{t}, U_{t} \in \tau_{K}$ such that $Q_{t}=P_{t} \cap K_{1}$ and $S_{t}=U_{t} \cap K_{1}$. Put $\Omega_{y}=\left\{U_{t} \mid t \in X_{1}\right\}$. It is not difficult to check that $\Omega_{y}$ is a cover of $X$. Since $X$ is $\theta$-regular, there exists $R \in \tau_{X}$ such that $x \in R$ and $\operatorname{cl}_{X} R$ can be covered by finitely many elements of $\Omega_{y}$. Let $t_{1}, t_{2}, \ldots, t_{m} \in X_{1}$ be such elements that $\operatorname{cl}_{X} R \subseteq \bigcup_{j=1}^{m} U_{t_{j}}$ and $T \in \tau_{k}$ such that $R=T \cap X$. We put

$$
P=\bigcap_{j=1}^{m} P_{t_{j}}, \quad U=\bigcup_{j=1}^{m} U_{t_{j}}, \quad V=U \cap T .
$$

We leave the reader to verify that $U, V, P$ satisfy the conditions (1)-(4). Now we put $M=Y \cup \operatorname{cl}_{X} X_{1}$ and

$$
W=\left[M \backslash \operatorname{cl}_{\mathrm{M}}(U \cap M)\right] \cup\left[(X \backslash M) \backslash \mathrm{cl}_{\mathrm{X}}(V \cap X)\right]
$$

We claim that $W \in \tau_{K}, y \in W$, and $W \cap V=\varnothing$. It is clear that $K_{1} \subseteq M$. Then $(X \backslash M) \cap$ $K_{1}=\varnothing$, which gives $W \cap K_{1}=K_{1} \cap\left[M \backslash \operatorname{cl}_{M}(U \cap M)\right] \in \tau_{K_{1}}$. Further, $M \cap X=\operatorname{cl}_{X} X_{1}$, $X \backslash M=X \backslash \mathrm{cl}_{\mathrm{X}} X_{1}$. Then $W \cap X=\left[\mathrm{cl}_{\mathrm{X}} X_{1} \backslash \mathrm{cl}_{\mathrm{M}}(U \cap M)\right] \cup\left[\left(X \backslash \mathrm{cl}_{\mathrm{X}} X_{1}\right) \backslash \mathrm{cl}_{\mathrm{X}}(V \cap\right.$ $X)]$. But the set $\operatorname{cl}_{X} X_{1} \backslash \operatorname{cl}_{M}(U \cap M)$ is open in $\operatorname{cl}_{X} X_{1}$ since $\operatorname{cl}_{X} X_{1}$ is a subspace of $M$. But $\operatorname{cl}_{X} X_{1}$ is also a subspace of $X$, so there is $S \in \tau_{X}$ such that $\operatorname{cl}_{X} X_{1} \backslash \operatorname{cl}_{M}(U \cap M)=$ $S \cap \operatorname{cl}_{X} X_{1}$. Then $W \cap X=\left(S \cap \operatorname{cl}_{X} X_{1}\right) \cup\left[\left(X \backslash \operatorname{cl}_{X} X_{1}\right) \backslash \operatorname{cl}_{X}(V \cap X)\right]$. On the other hand, from (3) it follows $\operatorname{cl}_{X} X_{1} \cap \operatorname{cl}_{X}(V \cap X) \subseteq M \cap c_{X}(V \cap X) \subseteq M \cap U \subseteq \operatorname{cl}_{M}(U \cap M)$. Then $\left(S \cap \operatorname{cl}_{\mathrm{X}} X_{1}\right) \cap \operatorname{cl}_{\mathrm{X}}(V \cap X)=\left(S \cap \operatorname{cl}_{\mathrm{X}} X_{1}\right) \cap\left[\operatorname{cl}_{\mathrm{X}} X_{1} \cap \mathrm{cl}_{\mathrm{X}}(V \cap X)\right] \subseteq\left[\operatorname{cl}_{\mathrm{X}} X_{1} \backslash \operatorname{cl}_{\mathrm{M}}(U \cap M)\right]$ $\cap \mathrm{cl}_{\mathrm{M}}(U \cap M)=\varnothing$. Then $W \cap X=\left[\left(S \cap \mathrm{cl}_{\mathrm{X}} X_{1}\right) \backslash \mathrm{cl}_{\mathrm{X}}(V \cap X)\right] \cup\left[\left(X \backslash \operatorname{cl}_{\mathrm{X}} X_{1}\right) \backslash \mathrm{cl}_{\mathrm{X}}(V \cap\right.$ $X)]=\left[\left(S \cap \operatorname{cl}_{X} X_{1}\right) \cup\left(X \backslash \operatorname{cl}_{X} X_{1}\right)\right] \backslash \operatorname{cl}_{X}(V \cap X)=\left[S \cup\left(X \backslash \operatorname{cl}_{X} X_{1}\right)\right] \backslash \operatorname{cl}_{X}(V \cap X) \in \tau_{X}$. Hence, $W \in \tau_{K}$. Now we will show that $y \in W$. Since $y \in Y$, it follows $y \in M$. We will check that $y \notin \mathrm{cl}_{\mathrm{M}}(U \cap M)$. It follows from (4) that $U \cap P \cap X_{1}=\varnothing$ and $U \cap P \cap Y=\varnothing$. Then also $U \cap P \cap \operatorname{cl}_{X} X_{1}=\varnothing$, since the case $p \in U \cap P \cap \operatorname{cl}_{X} X_{1}$ for some $p$ implies that $p \in U \cap P \cap X \in \tau_{X}$ and hence $(U \cap P \cap X) \cap X_{1} \neq \varnothing$, which is a contradiction. It follows $U \cap P \cap M=(U \cap P \cap Y) \cup\left(U \cap P \cap \mathrm{cl}_{X} X_{1}\right)=\varnothing$. But then $P \cap M \in \tau_{M}$ is a neighborhood of $y$ which does not meet $U \cap M$, so $y \notin \mathrm{cl}_{M}(U \cap M)$. Then $y \in W$. Finally, $W \cap V=\left[(V \cap M) \backslash \operatorname{cl}_{\mathrm{M}}(U \cap M)\right] \cup\left[(V \cap(X \backslash M)) \backslash \mathrm{cl}_{\mathrm{X}}(V \cap X)\right] \subseteq[(V \cap M) \backslash(U \cap$ $M)] \cup\left[(V \cap X) \backslash \operatorname{cl}_{X}(V \cap X)\right]=(V \backslash U) \cap M=\varnothing$. Hence, $\tau_{K}$ is a $\mathscr{C}$-acceptable topology on $K$ with respect to $X, Y$ which means that $\mathscr{C}\left(X_{1}\right) \succcurlyeq \mathscr{b}(X)$.

Conversely, let $X \cap Y=\varnothing$ and suppose that $X$ is compactificable by $Y$. We put $K_{1}=$ $X_{1} \cup Y$ with the topology induced from $K$. Let $\Omega_{1}$ be an open cover of $K_{1}$. For every $V \in \Omega_{1}$, there exists $U(V) \in \tau_{K}$ such that $V=U(V) \cap K_{1}$. We put $\Omega=\{U(V) \mid V \in$ $\left.\Omega_{1}\right\}$. Let $x \in K$. If $x \in K_{1}$, there is some $V \in \Omega_{1}$ such that $x \in V \subseteq U(V)$. Suppose that $x \in K \backslash K_{1}=X \backslash X_{1}$. Since $X_{1}$ is a minimal subspace of $X$, there is some $x_{1} \in X_{1}$ such that $x_{1} \in \operatorname{cl}_{\mathrm{X}}\{x\}$. There is $V \in \Omega_{1}$ such that $x_{1} \in V \subseteq U(V)$. But then $x \in U(V)$. Therefore, $\Omega$ is a cover of $K$. Since $K$ is compact, there exist $V_{1}, V_{2}, \ldots, V_{n}$ such that $K \subseteq \bigcup_{i=1}^{n} U\left(V_{i}\right)$, which implies that $K_{1} \subseteq \bigcup_{i=1}^{n} V_{i}$. Hence, $K_{1}$ is compact and $\tau_{K_{1}}$ obviously is a $\mathscr{C}$-acceptable 
topology on $K_{1}$. Then $X_{1}$ is compactificable by $Y$, which means that $\mathscr{C}(X) \succcurlyeq \mathscr{C}\left(X_{1}\right)$. Together with the result of the previous paragraph, we have $\mathscr{C}(X)=\mathscr{C}\left(X_{1}\right)$.

The continuation of this paper is the forthcoming paper "The compactificability classes of certain spaces" in which we will study the compactificability classes of some spaces constructed from the real line and Cantor or Tykhonov cubes.

\section{Acknowledgment}

This research is supported by the research intention of the Ministry of Education of the Czech Republic MSM0021630503 (MIKROSYN).

\section{References}

[1] R. Engelking, General Topology, PWN-Polish Scientific, Warsaw, Poland, 1977.

[2] A. Császár, General Topology, Akademiai Kiadó, Budapest, Hungary, 1978.

[3] D. S. Janković, " $\theta$-regular spaces," International Journal of Mathematics and Mathematical Sciences, vol. 8, no. 3, pp. 615-619, 1985.

[4] M. M. Kovár, "On $\theta$-regular spaces," International Journal of Mathematics and Mathematical Sciences, vol. 17, no. 4, pp. 687-692, 1994.

[5] H. Herrlich, "Compact $T_{0}$-spaces and $T_{0}$-compactifications," Applied Categorical Structures, vol. 1, no. 1, pp. 111-132, 1993.

[6] M. Hušek, "Čech-Stone-like compactifications for general topological spaces," Commentationes Mathematicae Universitatis Carolinae, vol. 33, no. 1, pp. 159-163, 1992.

[7] M. M. Kovár, "Which topological spaces have a weak reflection in compact spaces?" Commentationes Mathematicae Universitatis Carolinae, vol. 36, no. 3, pp. 529-536, 1995.

[8] M. M. Kovár, "Mutually compactificable topological spaces," to appear in International Journal of Mathematics and Mathematical Sciences.

[9] M. M. Kovár, "A remark on $\theta$-regular spaces," International Journal of Mathematics and Mathematical Sciences, vol. 21, no. 1, pp. 199-200, 1998.

Martin Maria Kovár: Department of Mathematics, Faculty of Electrical Engineering and Communication, University of Technology, Technická 8, 61669 Brno, Czech Republic Email address: kovar@feec.vutbr.cz 


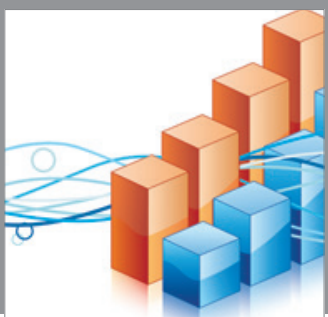

Advances in

Operations Research



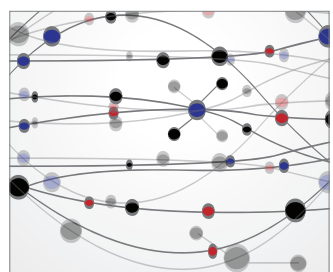

\section{The Scientific} World Journal
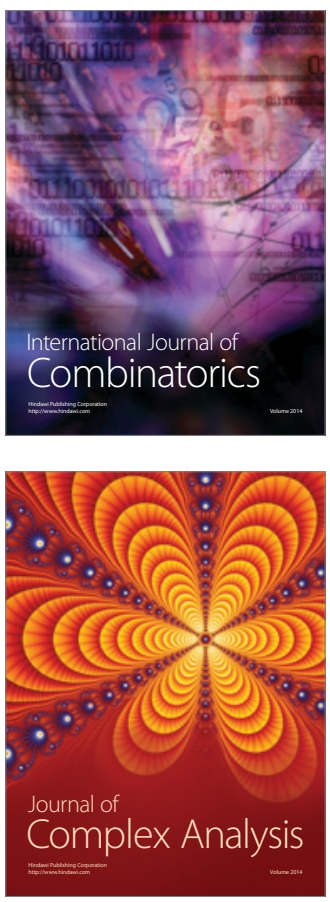

International Journal of

Mathematics and

Mathematical

Sciences
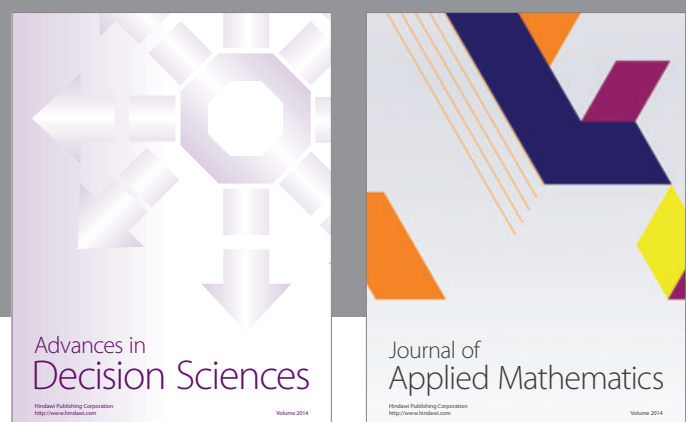

Journal of

Applied Mathematics
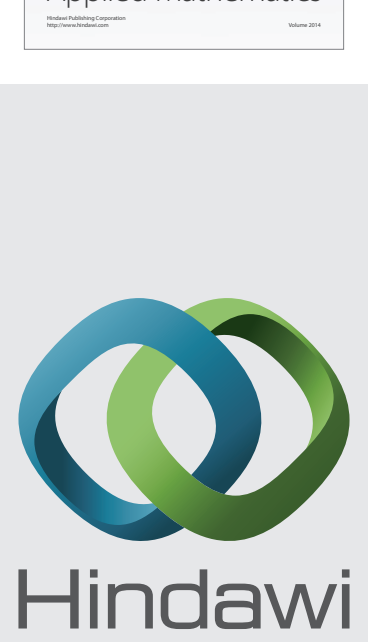

Submit your manuscripts at http://www.hindawi.com
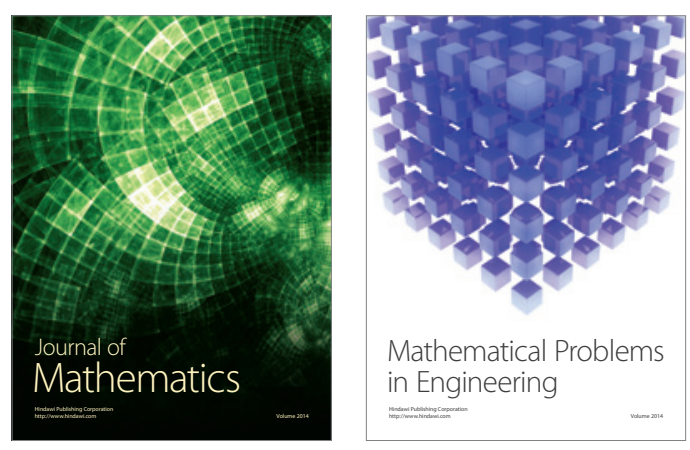

Mathematical Problems in Engineering
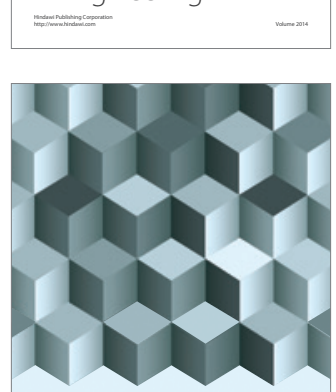

Journal of

Function Spaces


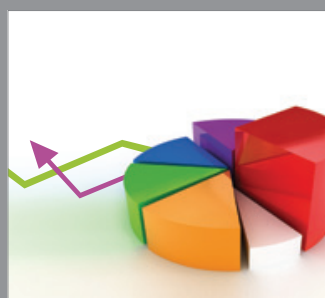

ournal of

Probability and Statistics

Promensencen
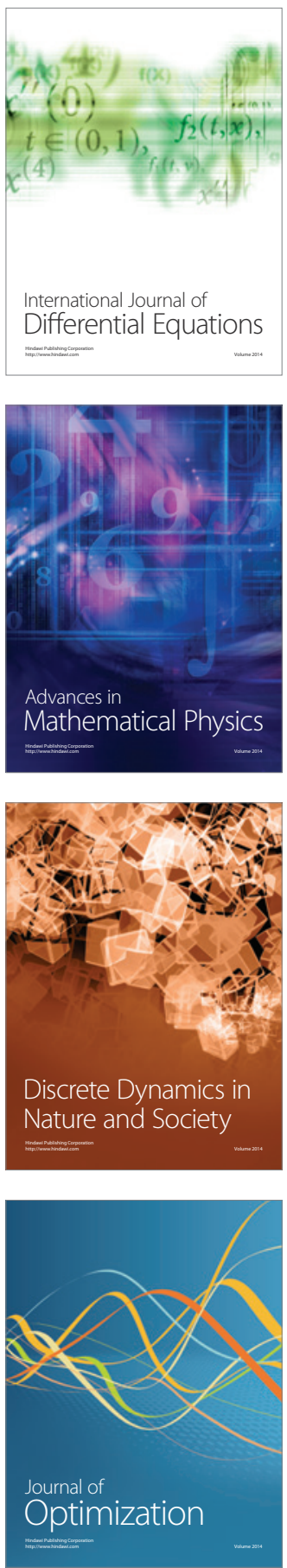